\title{
Cumulative Author Index
}

\section{Volume 4 (2015)}

Ambrosini, R., see Bolli, P.

Barsdell, B. R., see Kocz, J.

Bartolini, M., see Bolli, P.

Bernardi, G., see Kocz, J.

Bolli, P., Orlati, A., Stringhetti, L., Orfei, A., Righini, S., Ambrosini, R., Bartolini, M., Bortolotti, C., Buffa, F., Buttu, M., Cattani, A., D'Amico, N., Deiana, G., Fara, A., Fiocchi, F., Gaudiomonte, F., Maccaferri, A., Mariotti, S., Marongiu, P., Melis, A., Migoni, C., Morsiani, M., Nanni, M., Nasyr, F., Pellizzoni, A., Pisanu, T., Poloni, M., Poppi, S., Porceddu, I., Prandoni, I., Roda, J., Roma, M., Scalambra, A., Serra, G., Trois, A., Valente, G., Vargiu, G. P. and Zacchiroli, G., Sardinia Radio Telescope: General Description, Technical Commissioning and First Light

Bortolotti, C., see Bolli, P.

Bourke, S., see Kocz, J.

Buffa, F., see Bolli, P.

Buttu, M., see Bolli, P.

Cattani, A., see Bolli, P.

Clark, M. A., see Kocz, J.

Clarke, T. E., see Obenberger, K. S.

\author{
3\&4 (2015) 1550008 \\ 1\&2 (2015) 1550003 \\ 3\&4 (2015) 1550008 \\ 1\&2 (2015) 1550003
}

3\&4 (2015) 1550008

3\&4 (2015) 1550008

1\&2 (2015) 1550003

3\&4 (2015) 1550008

3\&4 (2015) 1550008

3\&4 (2015) 1550008

1\&2 (2015) 1550003

1\&2 (2015) 1550004
Craig, J., see Kocz, J.

D'Amico, N., see Bolli, P.

Deiana, G., see Bolli, P.

Dexter, M., see Kocz, J.

Dowell, J., see Kocz, J.

Dowell, J., see Obenberger, K. S.

Drysdale, T. D., see Johnson, B. R.

Dubois, A., see Obenberger, K. S.

Dubois, D., see Obenberger, K. S.

Eftekhari, T., see Kocz, J.

Ellingson, S., see Kocz, J.

Fara, A., see Bolli, P.

Fiocchi, F., see Bolli, P.

Fujikawa, S., see Johnson, B. R.

Fujishiro, N., see Kataza, H. Gaudiomonte, F., see Bolli, P.

Greenhill, L. J., see Kocz, J.

Hallinan, G., see Kocz, J.

Hartman, J., see Kocz, J.

Hartman, J. M., see Obenberger, K. S.

Henning, P. A., see Obenberger, K. S.

Hickish, J., see Magro, A.

Hull, C. L. H. and Plambeck, R. L., The $1.3 \mathrm{~mm}$ FullStokes Polarization System at $C A R M A$

Ikeda, Y., see Kataza, H. $\quad \mathbf{1 \& 2}$ (2015) 1550001

Jameson, A., see Kocz, J. $\quad \mathbf{1 \& 2}$ (2015) 1550003
1\&2 (2015) 1550005

1\&2 (2015) 1550003

3\&4 (2015) 1550008

3\&4 (2015) 1550008

1\&2 (2015) 1550003

1\&2 (2015) 1550003

1\&2 (2015) 1550004

3\&4 (2015) 1550007

1\&2 (2015) 1550004

1\&2 (2015) 1550003

1\&2 (2015) 1550003

3\&4 (2015) 1550008

3\&4 (2015) 1550008

3\&4 (2015) 1550007

1\&2 (2015) 1550001

3\&4 (2015) 1550008

1\&2 (2015) 1550003

1\&2 (2015) 1550003

1\&2 (2015) 1550003

1\&2 (2015) 1550004

1\&2 (2015) 1550004

1\&2 (2015) 1550002
1\&2 (2015) 1550004 
Johnson, B. R., Vourch, C. J., Drysdale, T. D., Kalman, A., Fujikawa, S., Keating, B. and Kaufman, J., A Cubesat for Calibrating Ground-Based and SubOrbital Millimeter-Wave Polarimeters (CalSat)

Kalman, A., see Johnson, B. R.

Kataza, H., Sakon, I., Wada, T., Sarugaku, Y., Fujishiro, N., Ikeda, Y., Mitani, S., Ohyama, Y. and Kobayashi, N., Performance Estimation of the Mid-Infrared Camera and Spectrometer Aboard SPICA

Kaufman, J., see Johnson, B. R.

Keating, B., see Johnson, B. R.

Kobayashi, N., see Kataza, H. Kocz, J., Greenhill, L. J., Barsdell, B. R., Price, D., Bernardi, G., Bourke, S., Clark, M. A., Craig, J., Dexter, M., Dowell, J., Eftekhari, T., Ellingson, S., Hallinan, G., Hartman, J . , J a m e s o n, A ., MacMahon, D., Taylor, G., Schinzel, F. and Werthimer, D., Digital Signal Processing Using Stream High Performance Computing: A 512-Input Broadband Correlator for Radio Astronomy

Lazio, J., see Obenberger, K. S.

Maccaferri, A., see Bolli, P. MacMahon, D., see Kocz, J.

Magro, A., Zarb Adami, K. and Hickish, J., GPU-Powered Coherent Beamforming

Mariotti, S., see Bolli, P.

Marongiu, P., see Bolli, P. Melis, A., see Bolli, P.
3\&4 (2015) 1550007

3\&4 (2015) 1550007

1\&2 (2015) 1550001

3\&4 (2015) 1550007

3\&4 (2015) 1550007

1\&2 (2015) 1550001

1\&2 (2015) 1550003

1\&2 (2015) 1550004

3\&4 (2015) 1550008

1\&2 (2015) 1550003

1\&2 (2015) 1550002

3\&4 (2015) 1550008

3\&4 (2015) 1550008

3\&4 (2015) 1550008
Michalak, S., see Obenberger, K. S.

Migoni, C., see Bolli, P. Mitani, S., see Kataza, H. Morsiani, M., see Bolli, P. Nanni, M., see Bolli, P. Nasyr, F., see Bolli, P. Obenberger, K. S., Taylor, G. B., Hartman, J. M., Clarke, T. E., Dowell, J., Dubois, A., Dubois, D., Henning, P. A., Lazio, J., Michalak, S. and Schinzel, F. K., Monitoring the Sky with the Prototype All-Sky Imager on the $L W A 1$

Ohyama, Y., see Kataza, H. Orfei, A., see Bolli, P.

Orlati, A., see Bolli, P. Pellizzoni, A., see Bolli, P. Pisanu, T., see Bolli, P. Plambeck, R. L., see Hull, C. L. H.

Poloni, M., see Bolli, P. Poppi, S., see Bolli, P. Porceddu, I., see Bolli, P. Prandoni, I., see Bolli, P. Price, D., see Kocz, J. Righini, S., see Bolli, P. Roda, J., see Bolli, P. Roma, M., see Bolli, P. Sakon, I., see Kataza, H. Sarugaku, Y., see Kataza, H. Scalambra, A., see Bolli, P. Schinzel, F., see Kocz, J.

Schinzel, F. K., see Obenberger, K. S. Serra, G., see Bolli, P. Shukla, P., see Thesiya, D. Srinivas, A. R., see Thesiya, D.

Stringhetti, L., see Bolli, P. Taylor, G., see Kocz, J.

Taylor, G. B., see Obenberger, K. S.

Thesiya, D., Srinivas, A. R. and Shukla, P., A Novel Lateral Deployment Mechanism for Segmented Mirror/Solar Panel of Space Telescope
$1 \& 2$ (2015) 1550004

3\&4 (2015) 1550008

1\&2 (2015) 1550001

3\&4 (2015) 1550008

3\&4 (2015) 1550008

3\&4 (2015) 1550008

1\&2 (2015) 1550004

1\&2 (2015) 1550001

3\&4 (2015) 1550008

3\&4 (2015) 1550008

3\&4 (2015) 1550008

3\&4 (2015) 1550008

1\&2 (2015) 1550005

3\&4 (2015) 1550008

3\&4 (2015) 1550008

3\&4 (2015) 1550008

3\&4 (2015) 1550008

1\&2 (2015) 1550003

3\&4 (2015) 1550008

3\&4 (2015) 1550008

3\&4 (2015) 1550008

1\&2 (2015) 1550001

1\&2 (2015) 1550001

3\&4 (2015) 1550008

$1 \& 2$ (2015) 1550003

1\&2 (2015) 1550004

3\&4 (2015) 1550008

3\&4 (2015) 1550006

3\&4 (2015) 1550006

3\&4 (2015) 1550008

$1 \& 2$ (2015) 1550003

1\&2 (2015) 1550004

3\&4 (2015) 1550006 
Trois, A., see Bolli, P.

Valente, G., see Bolli, P.

Vargiu, G. P., see Bolli, P.

Vourch, C. J., see Johnson, B. R.

Wada, T., see Kataza, H.
3\&4 (2015) 1550008

3\&4 (2015) 1550008

3\&4 (2015) 1550008

3\&4 (2015) 1550007

1\&2 (2015) 1550001
Werthimer, D., see Kocz, J.

1\&2 (2015) 1550003

Zacchiroli, G., see Bolli, P. $\quad 3 \& 4$ (2015) 1550008 Zarb Adami, K., see Magro, A.

1\&2 (2015) 1550002 